\title{
Infrastructure Needs for Translational Integration of Mouse and Human Trials
}

\author{
John G. Clohessy ${ }^{1}$ and Elisa de Stanchina ${ }^{2,3}$ \\ ${ }^{1}$ Preclinical Murine Pharmacogenetics Facility, Division of Genetics, Department of Medicine, Beth Israel \\ Deaconess Cancer Center, Beth Israel Deaconess Medical Center, Dana-Farber/Harvard Cancer Center, Harvard \\ Medical School, Boston, Massachusetts 02215; ${ }^{2}$ Antitumor Assessment Core Facility, Molecular Pharmacology and \\ Chemistry Program, Memorial Sloan-Kettering Cancer Center, New York, New York 10065
}

\begin{abstract}
Advances in the treatment of human cancer are frequently limited by the inability to test novel drugs and drug combinations in patients in a rapid and streamlined manner. Increasing data from the application of clinically relevant mouse models has highlighted the ability of preclinical trials in mice to address this problem, and has paved the way for what is now termed the "Co-Clinical Trial Project," in which mouse trials are performed concurrently with human trials. This in turn enables efficient patient stratification and therapy optimization based on molecular determinants for effective treatment of cancer. To fully realize the potential of preclinical, coclinical, and postclinical trials in mice, there is a need to establish key principles for carrying out therapeutic mouse trials, to standardize practices for performing such trials, and to establish mouse hospitals where trials can be integrated with corresponding clinical trial efforts in humans. Here we describe critical infrastructural components that are required for effective implementation of such efforts and suggest a model for how mouse hospitals for clinical trials should be established.
\end{abstract}

Pioneering efforts to faithfully model human cancer are now showing promise as important tools to rapidly evaluate cancer therapies and predict clinical response based on molecular characteristics that are observed in human cancers (Regales et al. 2009; Nardella et al. 2011; Chesi et al. 2012). Studies that have established the effectiveness of this approach have recently led to the evolution of both coclinical and postclinical projects (Nardella et al. 2011; Chen et al, 2012; see also Lunardi et al. 2013).

Although the concept of preclinical trials is a very familiar one, whereby in vitro or in vivo models are used to evaluate the therapeutic potential of a novel drug treatment, the concept of post- and coclinical trials are less well established, and are elaborated on in Introduction: Effective Utilization and Appropriate Selection of Genetically Engineered Mouse Models for Translational Integration of Mouse and Human Trials (Abate-Shen and Pandolfi 2013). In the postclinical setting, clinical samples and data are used to retrospectively stratify patients based on molecular and genetic lesions uncovered by going back and reanalyzing samples as a consequence of more recent discoveries. Most recently, the concept of coclinical trials has entered the arena and aims to carry out in vivo mouse trials concurrently with human clinical trials to allow for development of a more personalized approach to the treatment of disease.

\footnotetext{
${ }^{3}$ Correspondence: destance@mskcc.org

(c) 2013 Cold Spring Harbor Laboratory Press

Cite this introduction as Cold Spring Harb Protoc; 2013; doi:10.1101/pdb.top078782
} 
J.G. Clohessy and E. de Stanchina

To facilitate the adoption of a consistent approach in the design and implementation of mouse clinical trials, the Mouse Models for Human Cancer Consortium (MMHCC), an NCI collaborative program dedicated to the application of mouse models in cancer research, proposes to develop standard operating procedures (SOPs) for the care, treatment, and study of mice in pre-, co-, and postclinical settings. The objective of the MMHCC is to develop a framework that represents "standard of care" for mouse studies across multiple centers to eliminate variability that arises from differences due to practice, while also developing a standard paradigm that can be adopted by centers wishing to partake in mouse clinical studies. The Infrastructure SOP will set out the basic physical and organizational structures and facilities that need to be in place to allow for Research Validated Best Practice and Field Tested Best Practice programs to be implemented, so as to provide recognized "standard of care" in mouse studies. To effectively mirror and inform trials in human patients, therapeutic trials in mice need to coordinate mouse treatment, imaging, and analysis concurrently and in a standardized manner. Curation of all data is also of great importance, and the deposition of such data in a central repository is imperative, such that all elements can be integrated and compared with patient responses in the relevant clinical trials. In addition, it is vitally important that scientists carrying out trials in mice and clinicians responsible for human trials coordinate efforts to ensure that mouse trials effectively mimic treatment plans used in human patients, especially with regard to treatment schedule, clinical observations, and outcome assessment. These steps will ensure that preclinical studies testing the efficacy of a potential therapy are designed in a rigorous way, so as to be informative, reproducible, and relevant to the clinical setting. Here we describe several key infrastructural elements that represent the foundations on which to build a successful approach to carry out mouse trials that can effectively inform the treatment of human cancers.

Key to ensuring an effective murine clinical trial program is the concept of the "Mouse Hospital" (Fig. 1), which facilitates the integration of pre- and coclinical trials in mice through the coordination of efforts from multiple facilities, including husbandry operation, veterinary services, pharmacy, imaging, analytical chemistry, pathology, biostatistics, and information technology (IT).

In establishing an infrastructure to house a functional mouse hospital that will carry out clinical trials, key areas that need to be considered include the physical space within which mice are housed and the housing environment (including caging conditions, cage ventilation, day/night cycles, etc.), with easy access to relevant imaging (positron emission tomography (PET), magnetic resonance imaging (MRI), bioluminescence, etc.) and procedural suites within a single facility. Procedures and standards need to be established and implemented to ensure that mice are housed in a pathogen and endo-/ectoparasite-free environment, with standard room temperature, humidity, air pressure differential, and day-night cycles adhered to and with food and water requirements.

Because of the different aspects involved in running any type of preclinical trial, coordination of each aspect of the experimental plan is of outmost importance to the success and reproducibility of the entire study. Key personnel in the Mouse Hospital should be in charge of this task, while specialized personnel should administer drugs in addition to performing clinical and efficacy assessments. Furthermore, veterinarians familiar with the specific study design should also be available in case of unexpected occurrences.

Access to drugs, and in particular the access to drugs already approved for use in the clinic, represents an important component of coclinical efforts and often constitutes a significant challenge, both in terms of access to large amounts of high-quality material and the cost of its production (Box 1). Although the direct purchase of drugs approved for use in the clinic honors patent provisions, for 


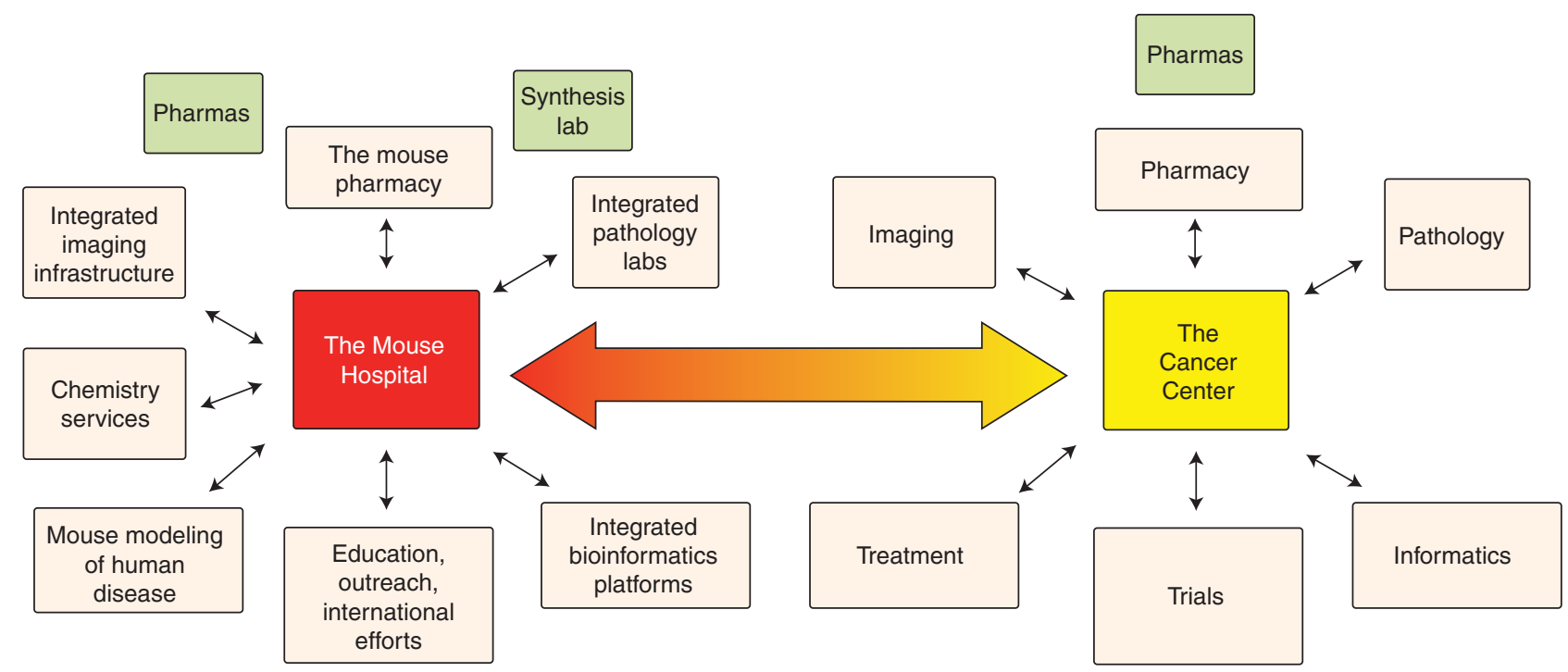

FIGURE 1. Infrastructure for the Mouse Hospital.

those drugs not already approved, the outsourcing of drug synthesis and production to a common facility can help with the provision of large amounts of drug at a reasonable cost and will ensure that all centers have access to material of the same quality.

Of note, early-generation drugs are often difficult to work with, because many of them can be hard to formulate, thereby limiting bioavailability. In the absence of standardized procedures for preparation and administration, a significant variability in efficacy may be observed among different centers. Therefore, the mouse pharmacy should not only take responsibility for distributing the compounds, but should also ensure that standardized procedures for drug preparation, administration, and storage are in place.

For drugs to be easily shared among centers participating in coclinical trials, a standardized material transfer agreement (MTA) template should also be put in place. The document should be drafted in such a way that it can be readily adapted and amended by multiple institutions, to not only allow for distribution of clinically relevant drugs, but to allow for such drugs to be used in multiple combinations, as determined by disease genetics in each clinical setting.

\section{BOX 1. ELEMENTS FOR CONSIDERATION WHEN DEVELOPING A PHARMACY FOR THE MOUSE HOSPITAL}

- Drug procurement In-house synthesis laboratory Outsourcing Pharmaceutical companies Branded versus generic compounds

- Quality control

- Formulation

- Vialing/labeling/dispensation

- Instructions for drug dosage, administration and storage, and safety precautions

- MTA forms 
To ensure that the clinical efforts in mice are consistent across multiple platforms, and in multiple centers, it is crucial to have SOPs in place for all activities related to each clinical trial (Box 2). In fact, even small changes in procedures may lead to discrepancies and thus difficulties in data comparison. For example, differences in enzymes, electrolytes, and hemolysis levels in mouse serum samples may simply be linked to the use of different blood collection and anesthesia methods (Traslavina et al. 2010). Thus, the aim of these SOPs is to ensure that trials are performed in a consistent, uniform, reliable, and reproducible manner across multiple independent centers.

Although implementation of strict Good Laboratory Practice guidelines (21CFR58) for these types of studies may be too onerous and therefore not feasible, the adherence to well-defined SOPs will provide a quality assurance for all work being performed. In addition, there should be significant congruence between SOPs in mouse and human trials. For example, ideally the same strategies and SOPs used in patient imaging should be applied to mouse trials, so as to allow for comparison of images from experiments in different models and/or laboratories. Assessment of pharmacokinetic and pharmacodynamic profiles is also commonly associated with clinical trials. Standardized sampling time points are chosen to reflect the human clinical trial setting, and SOPs (blood collection method, blood volume, protein extraction, LC/MS [liquid chromatography-mass spectrometry] method, data analysis software) need to be consistently followed to assure reproducibility.

Additionally, histopathology of organs and tissues of interest is commonly performed at time of kill as part of terminal readout analysis. Once again, it is of paramount importance that samples are handled in a consistent fashion, following standardized protocols for euthanasia, dissection, tissue fixation, staining, and histology interpretation.

Thus, there is a need for SOP development to facilitate and guide (i) the setup and establishment of mouse trials, particularly in a coclinical context, (ii) the treatment procedures themselves, (iii) the collection and analysis of treatment response, and (iv) the integration and reporting of data collected.

\section{EXPERIMENTAL DESIGN AND TREATMENT PROTOCOLS-ADVICE AND GUIDANCE!}

Once the animal models have been selected for a particular pre-, co-, or postclinical trial, it is important to define criteria for age, sex, and sample size (see also Introduction: Effective Utilization and Appropriate Selection of Genetically Engineered Mouse Models for Translational Integration of Mouse and Human Trials [Abate-Shen and Pandolfi 2013]). Sample size should be carefully chosen, so as to make sure that the study is designed with sufficient statistical power to address all questions relevant to the trial. Of note, an additional number of mice needs to be included to account for the fact that loss of mice is to be expected, especially in protocols that may include prolonged treatments with

BOX 2. IMPORTANT COMPONENTS IN THE DESIGN OF EFFECTIVE MOUSE TRIALS FOR TRANSLATIONAL STUDIES

- Establishment of detailed study protocols, in collaboration with scientists and clinicians

- Amendment of animal protocols to cover each new study

- Assignment of specialized clinical trial technicians to each protocol

- Coordination of services with husbandry, pharmacy, imaging, analytical chemistry, pathology, etc.

- SOP writing and maintenance

- Training of clinical trials technicians 
a combination of different chemotherapeutic agents or sequential imaging sessions. Animals are then randomized and assigned to the different treatment groups.

Although SOPs for drug formulation, storage, and stability will be put in place to ensure uniform efficacy, the drug dose and administration schedule should be chosen to mimic the corresponding human trial. Particular attention has to be paid to the time of drug administration, which has to be maintained constant, because drugs may have differential effects depending on the time of daily dosing and the impact of circadian rhythm on mouse metabolism (Innominato et al. 2010).

In addition, clinical and outcome assessments will have been defined at the time of study design and should be based on three main criteria: objectivity, reproducibility, and relevance to the corresponding human clinical trial. For example, treatment outcome in patients is often monitored by anatomical imaging (MRI, PET); therefore, this should also be the case for coclinical trials in the corresponding mouse cancer models. In this context, the time line for imaging should be defined ahead of time to mimic the human clinical study.

Body weights should be closely monitored and recorded daily along with other relevant clinical observations. Blood samples may be collected at predetermined intervals for hematology and clinical chemistry analysis, with considerations for collection methods as outlined above.

\section{DATA INTEGRATION AND COCLINICAL EVALUATIONS}

As already mentioned above, for mouse trials to inform human clinical trials in a real-time manner, integrated comparisons of mouse data need to be made rapidly available to investigators leading clinical trials in human patients (Box 3). Ideally, data should be coordinated in a centralized database by organ, and investigators should be able to organize and sort experiments according to specific treatments or specific genetic lesions. This would allow clinical investigators to rapidly evaluate efficacy for a specific drug or drug combination on multiple genetic lesions for that particular organ in mice, and vice versa. Additionally, information pertinent to treatment protocols, monitoring of disease, toxicity, etc. relating to these mouse trials should be available and easily accessible by clinical investigators. Of course, although data relating to mouse trials can be made freely available to all investigators, greater consideration needs to be taken with patient data, to maintain patient privacy. This is particularly relevant for the coclinical setting, perhaps more so than for pre- or postclinical trials. Access to sensitive patient information needs to be carefully regulated, and although patient

BOX 3. INFRASTRUCTURE CONSIDERATIONS FOR THE CURATION OF MOUSE AND HUMAN COCLINICAL DATA

- Centralized data repository-easily organized by Organ Genetic status Therapeutic clinical trial/drug

- Data made available Treatment protocols Imaging/pharmacokinetics/histopathology data Toxicity Efficacy Overview of clinical data (de-identified)

- Ability to cross-reference and compare different tissues and drug combinations

- Links and references to SOPs and other centers

- Permissions/data access Mouse data versus human data 
J.G. Clohessy and E. de Stanchina

response to treatment will inform the design of mouse trials, various security options should be considered to de-identify patients and maintain integrity of human data. Additionally, restricting access to such information to designated IP addresses or the use of a two-step verification, requiring a username and password to sign-in, followed by a verification code sent to a mobile device owned by a user, may also help ensure that unauthorized access to sensitive information does not occur. These are issues that may also now require careful consideration by IRB committees before approval of integration and storage of human data in this manner.

\section{EDUCATION AND TRAINING}

In establishing clinical ventures in mice, education and training are essential to instituting and maintain standards of practice. The development and implementation of training programs to ensure all participating personnel are properly trained and instructed in relevant procedures and practices is integral to achieving uniformity and reproducibility in these efforts across multiple platforms and institutes. Such programs can also greatly facilitate the divides that exist between basic scientists and clinicians, bridging knowledge gaps, and fostering open communication, ultimately leading to more focused efforts.

\section{THE FUTURE OF MOUSE CLINICAL STUDIES}

As can be appreciated from this short summary, the infrastructure required to carry out clinical studies in mice is very substantial, and in fact does not differ much from that of a cancer hospital. However, the implementation of guidelines for Research Validated Best Practice and Standard of Care in pre-, co-, or postclinical experiments should help reduce issues of variability and reproducibility. Implementation of this rigorous approach will likely speed up drug development but more importantly it should result in a greater ability to translate discovery into clinical success. It is hoped that the adoption of such a model to complement these types of clinical studies will significantly improve the efficacy of cancer treatments and truly enable a more personalized and successful approach to the treatment and eradication of cancer.

\section{REFERENCES}

21CFR58 - Code of Federal Regulations Title 21—Food and Drugs. Chapter I: Food and Drug Administration. Department of Health and Human Services. Subchapter A: General. Part 58 Good Laboratory Practices for Nonclinical Laboratory Studies. U.S. Food and Drug Administration. Revised as of April 1, 2012. http://www.accessdata.fda.gov/scripts/cdrh/ cfdocs/cfCFR/CFRSearch.cfm?CFRPart=58\&showFR=1.

Abate-Shen C, Pandolfi PP. 2013. Effective utilization and appropriate selection of genetically engineered mouse models for translational integration of mouse and human trials. Cold Spring Harb Protoc doi: 10.1101/pdb.top078774.

Chen Z, Cheng K, Walton Z, Wang Y, Ebi H, Shimamura T, Liu Y, Tupper T, Ouyang J, Li J, et al. 2012. A murine lung cancer co-clinical trial identifies genetic modifiers of therapeutic response. Nature 483: 613-617. doi: 10.1038 /nature10937.

Chesi M, Matthews GM, Garbitt VM, Palmer SE, Shortt J, Lefebure M, Stewart AK, Johnstone RW, Bergsagel PL. 2012. Drug response in a genetically engineered mouse model of multiple myeloma is predictive of clinical efficacy. Blood 120: 376-385.
Innominato PF, Lévi FA, Bjarnason GA. 2010. Chronotherapy and the molecular clock: Clinical implications in oncology. Adv Drug Deliv Rev 62: 979-1001.

Lunardi A, Ala U, Epping MT, Salmena L, Clohessy JG, Webster KA, Wang G, Mazzucchelli R, Bianconi M, Stack EC, et al. 2013. A co-clinical approach identifies mechanisms and potential therapies for androgen deprivation resistance in prostate cancer. Nat Genet 45: 747- 755.

Nardella C, Lunardi A, Patnaik A, Cantley LC, Pandolfi PP. 2011. The APL paradigm and the "co-clinical trial" project. Cancer Discov 1: 108116.

Regales L, Gong Y, Shen R, de Stanchina E, Vivanco I, Goel A, Koutcher JA, Spassova M, Ouerfelli O, Mellinghoff IK, et al. 2009. Dual targeting of EGFR can overcome a major drug resistance mutation in mouse models of EGFR mutant lung cancer. J Clin Invest 119: 30003010.

Traslavina RP, King EJ, Loar AS, Riedel ER, Garvey MS, Ricart-Arbona R, Wolf FR, Couto SS. 2010. Euthanasia by $\mathrm{CO}_{2}$ inhalation affects potassium levels in mice. J Am Assoc Lab Anim Sci 49: 316-322. 


\section{Infrastructure Needs for Translational Integration of Mouse and Human Trials}

John G. Clohessy and Elisa de Stanchina

Cold Spring Harb Protoc; doi: 10.1101/pdb.top078782 originally published online September 30, 2013

\begin{tabular}{rc}
\hline $\begin{array}{c}\text { Email Alerting } \\
\text { Service }\end{array}$ & Receive free email alerts when new articles cite this article - click here. \\
\hline $\begin{array}{c}\text { Subject } \\
\text { Categories }\end{array}$ & $\begin{array}{c}\text { Browse articles on similar topics from Cold Spring Harbor Protocols. } \\
\text { Mouse (437 articles) }\end{array}$ \\
\hline
\end{tabular}

